\title{
Schizophrenia in celiac disease: a myth or reality?
}

\author{
Raffaella Mormile $^{1}$
}

Accepted: 25 October 2015 /Published online: 31 October 2015

(C) Springer-Verlag Berlin Heidelberg 2015

\section{Dear Editor:}

Schizophrenia (SCZ) is a devastating neuropsychiatric disorder. The prevalence of SCZ is consistently about $1 \%$ throughout the world. It characteristically begins in late adolescence or young adulthood. Both environmental and genetic factors have been reported to influence the risk for developing SCZ. However, the exact cause of SCZ is not currently known. It has been proposed that aberrant autoimmune responses may contribute to the development of SCZ. It has been advised that schizophrenic subjects show higher $\mathrm{T}$ helper $1 / \mathrm{T}$ helper 2 (Th1/Th2) ratio. It has been highlighted that effective neuroleptic treatment provides benefits in terms of the imbalance between Th1 cytokines and Th2 cytokines in schizophrenic patients. Th1 cells are well established to be involved in autoimmune diseases. Th1 effector cells produce predominantly pro-inflammatory cytokines such as IFN- $\gamma$. Various cytokines have been observed in connection with SCZ, but the findings are controversial. Pro-inflammatory cytokines play pleiotropic roles in the central nervous system including neurogenesis as well as inflammatory responses. Neurogenesis represents the complex process of generating new neurons from neural stem or progenitor cells. It has been stated that neurogenesis may have an important role in the pathogenesis of SCZ supporting the association of inflammation and neurogenesis. Neurogenesis involves many steps such as integration of newly generated neurons into the existing functional neuronal cir-

Raffaella Mormile

raffaellamormile@alice.it

1 Division of Pediatrics and Neonatology, Moscati Hospital, Via A. Gramsci, 3, 81031 Aversa, Italy cuitry. Newly generated neurons in adulthood have been implicated in the development of SCZ. Interestingly, it has been shown that IFN- $\gamma$ signaling via signal transducer and activator 1 (STAT1) regulates neurogenesis in normal adult brain. STAT1 is activated by IFN- $\gamma$ mediating the effects induced by this cytokine. A number of autoimmune diseases have been shown in patients affected by SCZ. Among autoimmune disorders, celiac disease (CD) has been found for years in association with SCZ. CD is a lifelong autoimmune disorder occurring in genetically predisposed individuals where the ingestion of gluten leads to damage in the small intestine. Although patients with CD suffer primarily from gastrointestinal symptoms, $\mathrm{CD}$ may result in a variety of extra intestinal disorders. Similarly to SCZ, an aberrant Th1 immune reaction has been connected with the development of CD. IFN- $\gamma$ has been described as a key determinant in the pathogenesis of CD. Moreover, IFN- $\gamma$ gene polymorphisms have been linked to $C D$ susceptibility. STAT1 has been implicated in the pathogenesis of CD. It has been observed that STAT1 is an important feature of the inflammatory response in $\mathrm{CD}$. IFN- $\gamma$ has been described to contribute to the maintenance of chronic intestinal inflammation by regulating the expression and the activation of STAT1. Interestingly, increased serum levels of IFN- $\gamma$ have been constantly connected with hyperactivation of STAT1 in CD patients. Taken together into account, we suggest a causal link between CD and SCZ. We speculate that the risk for $\mathrm{SCZ}$ in $\mathrm{CD}$ patients may be dependent on the interplay between IFN- $\gamma$ and STAT1. We hypothesize that IFN- $\gamma$ gene polymorphisms may account for aberrant STAT1 expression resulting in disruption of neurogenesis. Thus, patients with $\mathrm{CD}$ should require neurological screening examination at the time of diagnosis and later in order to precociously identify patients at high risk of SCZ. 